\title{
Erratum to: An ecological risk assessment of heavy metal contamination in the surface sediments of Bosten Lake, northwest China
}

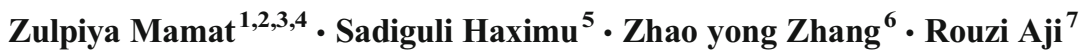

Published online: 19 February 2016

(C) Springer-Verlag Berlin Heidelberg 2016

Erratum to: Environ Sci Pollut Res

DOI 10.1007/s11356-015-6020-3

The original publication of this paper contains a mistake. In the Acknowledgments section, the fund number of "the Open Project of Xinjiang Key Laboratory of Lake Environment and Resources in Arid Zone, Xinjiang Normal University (X2014KL0106)", is not X2014KL0106 but should be 2014KL0106.

The online version of the original article can be found at http://dx.doi.org/ 10.1007/s11356-015-6020-3.

Zulpiya Mamat

zulpiya1219@163.com

1 Xinjiang Key Laboratory of Lake Environment and Resources in Arid Zone, Xinjiang Normal University, Urumqi 830054, China

2 Earthquake Administration of Xinjiang Uygur Autonomous Region, Urumqi 830000, China

3 College of Resources and Environment Science / Key Laboratory of Oasis Ecology (Ministry of Education), Xinjiang University, Urumqi 830046, China

4 Ecological Postdoctoral Research Station, Xinjiang University, Urumqi 830046, China

5 Editorial Department of Journal, Xinjiang Normal University, Urumqi 830054, China

6 College of Chemistry Environmental and Engineering, Shenzhen University, Shenzhen 518060, China

7 Xinjiang Meteorological Bureau, Urumqi 830002, China 